\title{
Emission properties of printed organic semiconductor lasers
}

\author{
Dario Pisignano, Luana Persano, Elisa Mele, Paolo Visconti, Roberto Cingolani, and Giuseppe Gigli \\ National Nanotechnology Laboratory of the Istituto Nazionale di Fisica della Materia, c/o Dipartimento di Ingegneria \\ dell'Innovazione, Università di Lecce, via Arnesano, I-73100 Lecce, Italy
}

Giovanna Barbarella and Laura Favaretto

Consiglio Nazionale delle Ricerche, Istituto pèr la Sintesi Organica è la Fotoreattiuità, Area della Ricerca di Bologna, via Gobetti 101, I-40129 Bologna, Italy

Received May 19, 2004

\begin{abstract}
We investigated the emission properties of a distributed-feedback resonator based on an organic semiconductor patterned by a novel printing technology. We observed the peak splitting of the photonic bandstructure of the periodic grating and extracted the effective refractive index of the outcoupled guided modes. The laser works at the second diffraction order, exhibiting narrow single-mode emission at $637 \mathrm{~nm}$, with a threshold as low as $37 \mu \mathrm{J} / \mathrm{cm}^{2}$. The results suggest that direct printing is a promising fabrication technique for optically confined integrated optoelectronics. (C) 2005 Optical Society of America

OCIS codes: $140.3390,140.3490$.
\end{abstract}

In recent years solid-state organic semiconductor lasers ${ }^{1}$ have attracted increasing interest because of the discovery of optical gain from a variety of conjugated compounds and in view of their future industrial application. Observations of high emission yield, photoluminescence (PL) line narrowing as a result of amplified spontaneous emission (ASE), ${ }^{2}$ and a large cross section for stimulated emission from molecular excited states $^{3,4}$ provide a clear indication that conjugated molecules can be used as active media in semiconductor lasers. Feedback for lasing can be obtained by use of different geometries, ${ }^{4-7}$ including distributed-feedback resonators (DFBs).

In particular, periodic variation of either the refractive index or the gain along the direction of wave propagation is the key element of $\mathrm{DFBs}^{8}$; the feedback is due to the Bragg diffraction by the corrugation, which continuously reflects part of the wave propagating in the forward direction of the medium. One-dimensional (1D) photonic crystals ${ }^{9}$ (PhCs) offer much more effective control of the device spectrum than external cavities, as the electron-photon coupling is directly affected by the optical mode density. For this reason, 1D PhCs lasers are strategically useful for the realization of electrically driven organic semiconductor lasers operating under steady-state excitation and high injection densities.

Today, novel mechanical lithographic approaches are able to transfer a pattern onto thin-film conjugated compounds with low cost, high fidelity, and good resolution. Among the various lithographies ${ }^{10,11}$ used to fabricate wavelength-scale periodic structures onto organic slabs, room-temperature nanoimprinting $^{12,13}$ (RT-NIL) offers advantages in dealing with light-emitting molecules. RT-NIL can be performed entirely in air without heating-induced incorporation of oxygen, which is responsible for heavy luminescence quenching in organic molecules. ${ }^{14}$ Dry printing of organic semiconductor lasers is currently possible, ${ }^{13}$ allowing one to develop a complete device by a single nanofabrication step.
In this work we achieve organic $1 \mathrm{D} \mathrm{PhC}$ resonators by use of RT-NIL. Si templates with a period of $\Lambda=400 \mathrm{~nm}$ and an area of $3.0 \mathrm{~mm} \times 7.0 \mathrm{~mm}$ were fabricated by electron-beam lithography (Fig. 1). Smaller masters $(2.0 \mathrm{~mm} \times 2.0 \mathrm{~mm})$ with the same period were also fabricated for $\mathrm{cw}$ optical measurements. The masters were used as molds for printing onto a thin film of a high-optical-gain (stimulated-emission cross section of $\left.6 \times 10^{-16} \mathrm{~cm}^{2}\right)^{4}$ thiophene-based pentamer ( $\mathrm{T} 5 \mathrm{oCx}$, inset of Fig. 2), spin cast onto quartz substrates. The patterned T5oCx $1 \mathrm{D}$ grating is shown in Fig. 2.

The photonic bandstructure of the DFBs was determined by photoexcitation of small-area samples by a HeCd laser $\left(\lambda=325 \mathrm{~nm}\right.$, excitation density $\left.0.1 \mathrm{~W} / \mathrm{cm}^{2}\right)$ and collection of the angle-resolved emission with a resolution of $1^{\circ}$. The large-area samples were pumped under vacuum ( $\cong 10^{-6}$ Torr) with a frequency-tripled $(\lambda=355 \mathrm{~nm}) \mathrm{Nd}$ :YAG laser delivering 3-ns-long pulses at $10 \mathrm{~Hz}$, with a cylindrical lens providing a rectangular excitation stripe of $7.0 \mathrm{~mm} \times 0.5 \mathrm{~mm}$.

The angle-resolved PL spectra of the printed organic semiconductor 1D PhCs under cw excitation are displayed in Fig. 3(a). A strong peak that is not present in the PL spectra of the untextured thin films is observable at $611 \mathrm{~nm}$ under normal collection (viewing angle $\Theta=0^{\circ}$ ), splitting into two separate peaks that shift along opposite directions as the detection is moved perpendicularly to the DFB grooves. For instance, at $\Theta=7^{\circ}$, the two peaks are almost $70 \mathrm{~nm}$

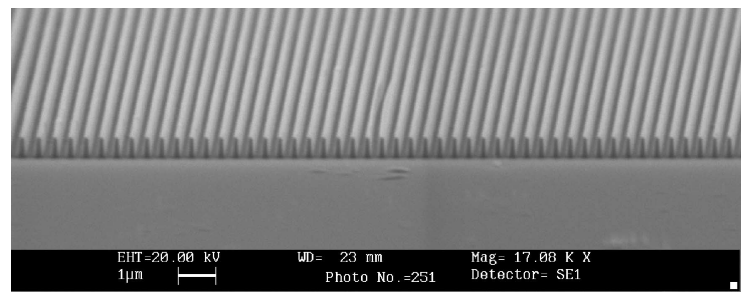

Fig. 1. Template gratings.

(C) 2005 Optical Society of America 


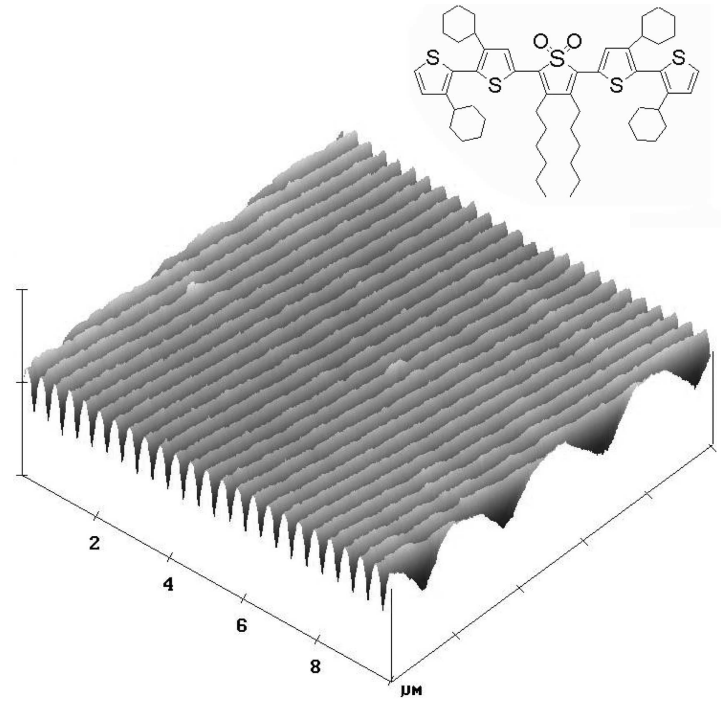

Fig. 2. Printed organic PhC. The features are $\sim 30 \mathrm{~nm}$ high. Inset, molecular structure of $\mathrm{T} 5 \mathrm{oCx}$.
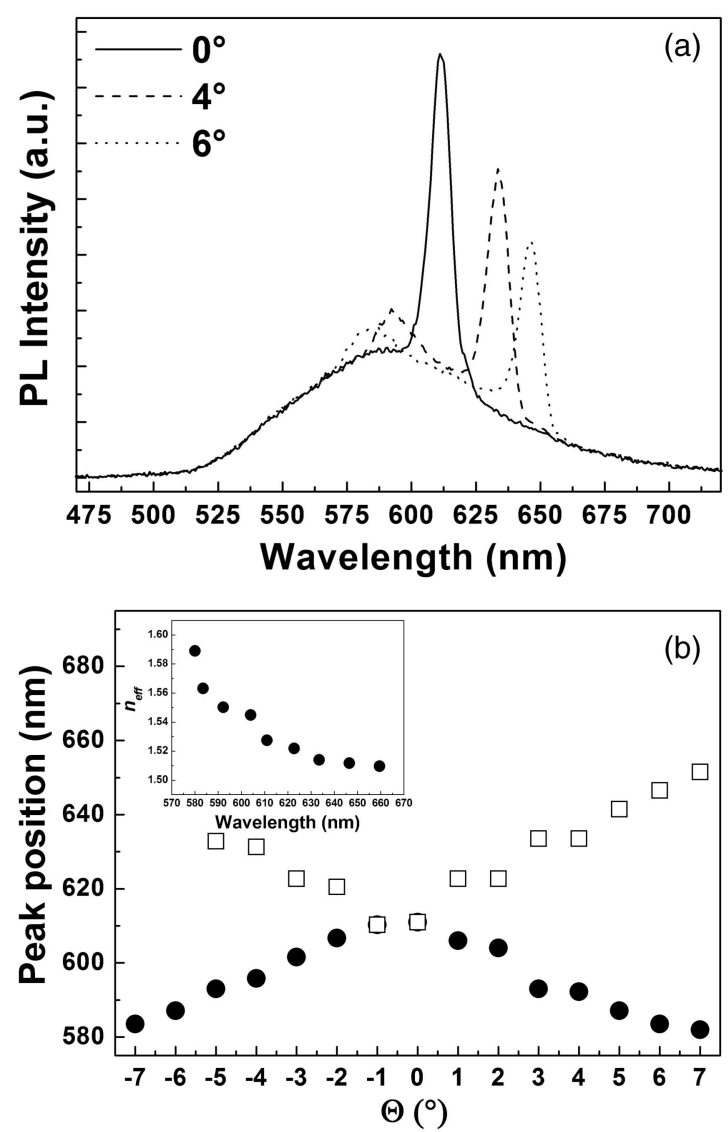

Fig. 3. (a) Normalized angle-resolved PL spectra of the printed 1D PhC. (b) Angular dependence of the emission peaks. Inset, wavelength dependence of $n_{\mathrm{eff}}$ of the Bragg-outcoupled guided modes.

apart, completely covering the ASE band of the T5oCx slab (580-660 nm). Coupling of guided optical modes of wavelength $\lambda$ and effective refractive index $n_{\text {eff }}$ with free radiation leaves the momentum at the interface between the organic film and the air unchanged; i.e., $\mathbf{k}_{0} \sin \Theta= \pm \mathbf{k}_{g} \pm n \mathbf{G}$, where $\mathbf{k}_{0}$ and $\mathbf{k}_{g}$ are the wave vectors of the free and the guided waves, respectively $\left(\left|\mathbf{k}_{0}\right|=2 \pi / \lambda\right.$ and $\left.\left|\mathbf{k}_{g}\right|=2 \pi n_{\text {eff }} / \lambda\right)$, $\mathbf{G}$ is the Bragg vector $(|\mathbf{G}|=2 \pi / \Lambda)$, and $n$ is an integer. This condition, namely, Bragg scattering of radiation moving along the forward and backward directions of the grating, ${ }^{15}$ determines the dependence of the peak emission wavelengths on the viewing angle [Fig. 3(b)].

From the angular dependence of the peak wavelength we extracted the effective refractive index of the outcoupled guided modes. $n_{\text {eff }}$ decreased from 1.59 to 1.51 as the wavelength increased from 580 to $660 \mathrm{~nm}$ [inset of Fig. 3(b)]. In our devices the optical confinement is provided by the surface periodicity at the $\mathrm{T} 5 \mathrm{oCx}$-air interface, which determines the spatial modulation of both the refractive index and the gain of the medium. The resulting emission, namely, the coupling of the bound optical modes with the free radiation, is related to the band structure of the $1 \mathrm{D} \mathrm{PhC}$, occurring mostly at the band edge corresponding to the lowest cavity losses. We note that the long-wavelength peak is more intense and narrower $(\mathrm{FWHM} \cong 8 \mathrm{~nm}$ ) than the short-wavelength peak $(\mathrm{FWHM} \cong 11 \mathrm{~nm})$. This indicates a higher finesse of the long-wavelength coupled mode, related to weaker output coupling, namely, to lower cavity losses. ${ }^{15}$ Therefore one expects that lasing action also takes place in the long-wavelength tail of the anticrossing bandstructure.

Finally, a large-area printed $\mathrm{T} 5 \mathrm{oCx}$ surface was employed as the corrugated interface of a DFB laser. The lasing wavelength, $\lambda_{m}$, is related to the cavity geometry by

$$
m \lambda_{m}=2 n_{\mathrm{eff}} \Lambda,
$$

where $m$ indicates the diffraction order onto the DFB grating. Organic-based DFB resonators have to date exploited mainly conjugated polymers and blends incorporating commercial dyes as active media. ${ }^{7,15,16}$ These compounds are usually deposited on previously patterned substrates, such as silica or transparent polymers, that can partially planarize the upper surface of the deposited gain film, thus weakening the overall feedback of the device. Direct imprinting ${ }^{13,17}$ can instead provide much more precise control of the organic-air interface.

The DFB operates at $637 \mathrm{~nm}$, exhibiting a narrow (FWHM $<1 \mathrm{~nm}$ ) single-mode laser emission (Fig. 4). The DFB mode oscillates in the long-wavelength spectral feature of the $\mathrm{T} 5 \mathrm{oCx}$, as expected. However, it is quite distant from the band-edge wavelength determined under cw excitation (near 610-620 nm). This could be due to the spatial modulation of optical gain that takes place under pulsed excitation, which could hinder a closer correlation between the $\mathrm{cw} \mathrm{PhC}$ band structure and the wavelength of laser emission. ${ }^{15}$ One should also consider the possibility of slight geometric differences (for example, in the duty cycle of the periodic grating and consequently in $n_{\text {eff }}$ ) among the different printed samples, because of alignment mismatch during printing.

According to Eq. (1), our devices work at an order $m=2$. The emission from $\mathrm{T} 5 \mathrm{oCx}$ that does not match 


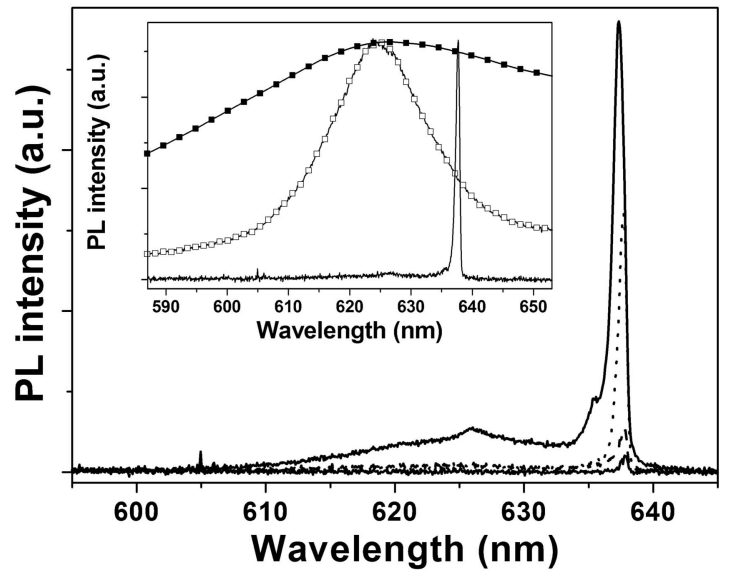

Fig. 4. DFB laser emission spectra at different absorbed excitation fluences (curves from bottom to top: 30, 50, 60, $80 \mu \mathrm{J} \mathrm{cm}^{-2}$ ). Inset, above-threshold laser spectrum (continuous curve), compared with the ASE (open squares) and $\mathrm{PL}$ (filled squares) spectra of T5oCx films.

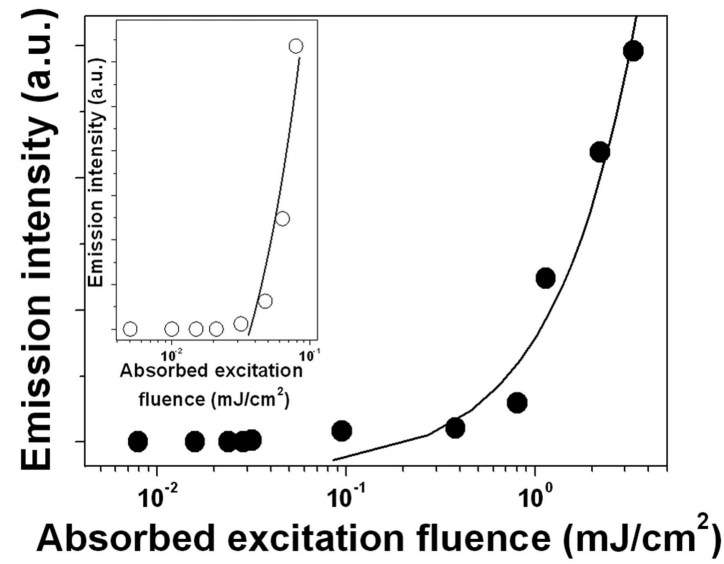

Fig. 5. Excitation fluence dependence of the DFB peak output intensity (device A). Inset, input-output characteristics of device B. The solid curves are linear fits to the experimental data (filled and open circles).

the resonating condition is almost completely quenched by the feedback of the device ${ }^{18}$ (the spontaneous PL and the ASE band of untextured T5oCx films are reported for comparison in the inset of Fig. 4). Above threshold, the DFB peak emission grows linearly with excitation (Fig. 5), and lasing thresholds occur at $220 \mu \mathrm{J} / \mathrm{cm}^{2}$ (device A, Fig. 5) and $37 \mu \mathrm{J} / \mathrm{cm}^{2}$ (device $\mathrm{B}$, inset of Fig. 5), depending on the imprinted sample. In particular, the threshold of laser B is almost four times smaller than that for third-order T5oCx DFBs (Ref. 13) and more than one order of magnitude lower than the pump density needed for the occurrence of ASE from a T5oCx planar slab, confirming the effectiveness of the distributed-feedback mechanisms.

Some issues related to RT-NIL need further investigation, including the ultimate achievable resolution and reproducibility. However, by printing the organic semiconductor $\mathrm{T} 5 \mathrm{oCx}$ we have demonstrated the great potential of this technique. Second-order DFB devices exhibited single-mode emission with a lasing threshold as low as $37 \mu \mathrm{J} / \mathrm{cm}^{2}$. We believe that the optical confinement of these gaining compounds and their direct dry printing deserve to be fully explored as a strategic route to the realization of organic-based electrically injected lasers.

D. Pisignano's e-mail address is dario.pisignano@ unile.it.

\section{References}

1. G. Kranzelbinder and G. Leising, Rep. Prog. Phys. 63, 729 (2000).

2. F. Hide, M. A. Díaz-García, B. J. Schwartz, M. R. Andersson, Q. Pei, and A. J. Heeger, Science 273, 1833 (1996).

3. W. Graupner, G. Leising, G. Lanzani, M. Nisoli, S. De Silvestri, and U. Scherf, Phys. Rev. Lett. 76, 847 (1996).

4. D. Pisignano, M. Anni, G. Gigli, R. Cingolani, M. Zavelani-Rossi, G. Lanzani, G. Barbarella, and L. Favaretto, Appl. Phys. Lett. 81, 3534 (2002).

5. T. Granlund, M. Theander, M. Berggren, M. Andersson, A. Ruzeckas, V. Sundstrom, G. Björk, M. Granström, and O. Inganäs, Chem. Phys. Lett. 288, 879 (2000).

6. S. V. Frolov, A. Fujii, D. Chinn, Z. V. Vardeny, K. Yoshino, and R. V. Gregory, Appl. Phys. Lett. 72, 2811 (1998)

7. M. Bergreen, A. Dodabalapur, and R. E. Slusher, Appl. Phys. Lett. 71, 2230 (1997).

8. H. Kolgelnik and V. Shank, Appl. Phys. Lett. 18, 152 (1971).

9. J. D. Joannopoulos, R. D. Meade, and J. N. Winn, Photonic Crystals: Molding the Flow of Light (Princeton U. Press, Princeton, N.J., 1995).

10. Y. Xia and G. M. Whitesides, Angew. Chem. Int. Ed. Engl. 37, 550 (1998).

11. S. Y. Chou, P. R. Krauss, and P. J. Renstrom, Science 272, 85 (1996).

12. D.-Y. Khang, H. Yoon, and H. H. Lee, Adv. Mater. 13, 749 (2001).

13. D. Pisignano, L. Persano, P. Visconti, R. Cingolani, G. Gigli, G. Barbarella, and L. Favaretto, Appl. Phys. Lett. 83, 2545 (2003).

14. M. Yan, L. J. Rothberg, F. Papadimitrakopoulos, M. E. Galvin, and T. M. Miller, Phys. Rev. Lett. 73, 744 (1994).

15. G. A. Turnbull, P. Andrews, M. J. Jory, W. L. Barnes, and I. D. W. Samuel, Phys. Rev. B 64, 125122 (2001).

16. S. Riechel, U. Lemmer, J. Feldmann, S. Berleb, A. G. Mückl, W. Brütting, A. Gombert, and V. Wittwer, Opt. Lett. 26, 593 (2001).

17. M. Gaal, C. Gadermaier, H. Plank, E. Moderreger, A. Pogantsch, G. Leising, and E. J. W. List, Adv. Mater. 15, 1165 (2003).

18. At high excitation densities $\left(\geq 0.8 \mathrm{~mJ} / \mathrm{cm}^{2}\right)$, damage to the imprinted nanostructure likely occurs as a result of pump-induced heating. This weakens the feedback along the slab, resulting in the rise of a significant spontaneous emission background (see, for instance, the highest spectrum in Fig. 4). 